\title{
Structural glass envelopes - Implementation of environmental studies into viscoelastic analysis
}

\author{
Lisa Rammig,b, Peter Lenk ${ }^{a, *}$ and Vladimir Marinov ${ }^{a}$ \\ ${ }^{a}$ Eckersley O'Callaghan, Kingsgate, London, UK \\ ${ }^{\mathrm{b}}$ Facade Research Group, Faculty of Architecture and the Built Environment, Delft Technical University
}

\begin{abstract}
The focus of this paper is to promote close collaborations of facade and structural engineers through integration of design services in order to deliver more optimal glass envelopes. To achieve the best performance, this process involves a careful selection of glass and coatings, radiation analysis to maximise daylight while controlling solar gain, and preventing overheating of spaces as well as careful detailing. Site-specific environmental data are used to accurately determine the performance requirements of the glass. Once the environmental impact is assessed the temperature in the interlayer is calculated over any chosen time domain. As part of the proposed process, a technique for determining the time-dependent structural behaviour of SGP laminated glass under varying temperature loads is also considered. In conclusion the authors examine the possibility of integration of both environmental and structural design approaches through the use of parametric software.
\end{abstract}

Keywords: Building envelopes, structural glass, parametric design, energy efficiency

\section{Introduction}

The increased need for more energy efficient envelopes is becoming the cornerstone for the design of many building structures and facades involving glass. Building envelope design becomes more complex due to increasingly stringent energy requirements as well as technological developments. Furthermore building envelope physics play an increasing role in the design and are today considered in great detail to ensure occupant comfort. As thermal comfort is dependent on many dynamic parameters these have to be analysed and balanced carefully to achieve a comfortable interior climate throughout the year. In addition, structural glass is increasingly becoming a starting point in the architectural exploration of envelopes. Almost always structural glass is employed as a laminate of two or more layers of glass, bonded with a plastic interlayer. Furthermore in certain design situations both energy and structural requirements need to be considered simultaneously. The scope of this study is to evaluate the potential for an increase in performance of structural glass through a combined site-specific environmental and structural analysis.

\footnotetext{
${ }^{*}$ Corresponding author: Peter Lenk, Eckersley O'Callaghan, Kingsgate, London, N19AE, UK. E-mail: peter@eckersleyo callaghan.com.
}

ISSN 2214-302X/14/\$35.00 @ 2014 - IOS Press and the authors. All rights reserved

This article is published online with Open Access and distributed under the terms of the Creative Commons Attribution Non-Commercial License. 


\section{Environmental parameters}

Due to the inherent characteristics of glazing, such as high thermal conductivity and transparency, the thermal impact on the interior space and as such on the thermal comfort, is much greater compared to other more solid materials like concrete or stone. As relates to the transparency, high performance coatings and external shading systems are commonly used to limit the amount of solar gain into the building and prevent overheating. The selection of these coatings and the type of glass determine the absorption coefficient of the overall build-up; the higher the absorption, the higher the temperature within the glass build-up. While glass temperature is a comfort parameter in itself, in this study it is used to inform the structural behaviour of glass elements. This is particularly important for laminated glass, as typically interlayers are made of viscoelastic materials, which properties also vary with temperature. For horizontal or inclined glazing this becomes an important consideration as the load transfer through the viscoelastic material decreases rapidly when exposed to different temperatures. This can lead to a large increase in glass deflections and stresses.

\subsection{Methodology}

With the thermal and radiation analyses of the building envelope based on location, orientation and exposure the temperature gradient through the glass build-up can be determined more precisely (Fig. 1, left). Rather than determining a set of peak temperature loads to design to, the proposed method uses site-specific weather data that allow the build-up temperature to be determined as a function of time.

Tools such as IES, Ecotect or DIVA for Rhino are used to carry out a radiation analysis on the building envelope. The volume of the envelope is exposed to the location specific solar radiation for a typical year, outputting radiation levels for certain intervals during this time. To ensure occupant comfort within an interior space several parameters have to be taken into account and set against each other to find the most optimal solution. Here these shall be defined as u-value, g-value, visible light transmission, surface temperature of the build-up and glare potential.

For the case study shown in Figure 1, right, several glass configurations have been specified and analysed under site-specific environmental conditions. For the analysis an Energy Plus weather file provided by the US department of Energy has been utilised (EnergyPlus, 2014). Surface temperatures as well as solar incident radiation in specific locations are calculated and then used in a further calculation to assess the temperature distribution throughout the glass build-up. Here peak loads
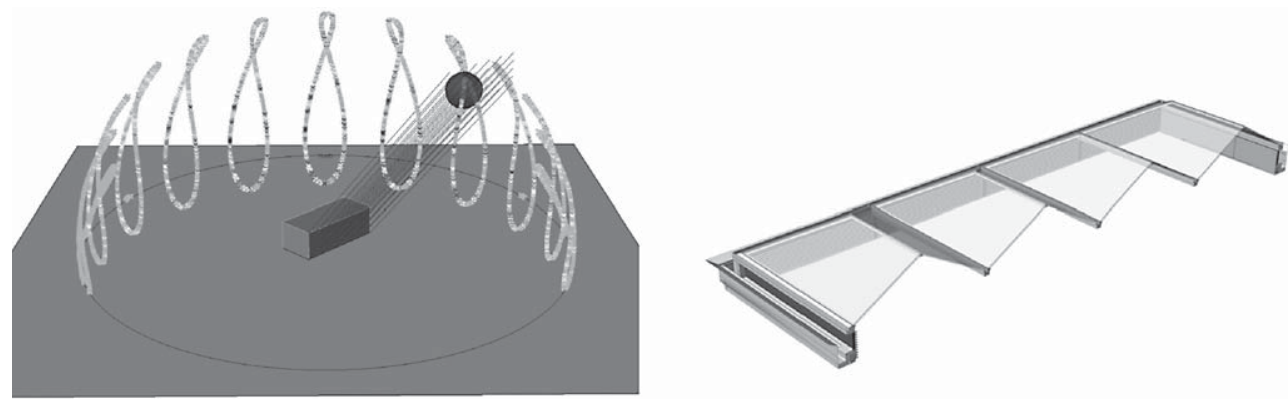

Fig. 1. Total solar radiation in IES (left), roof geometry (right). 

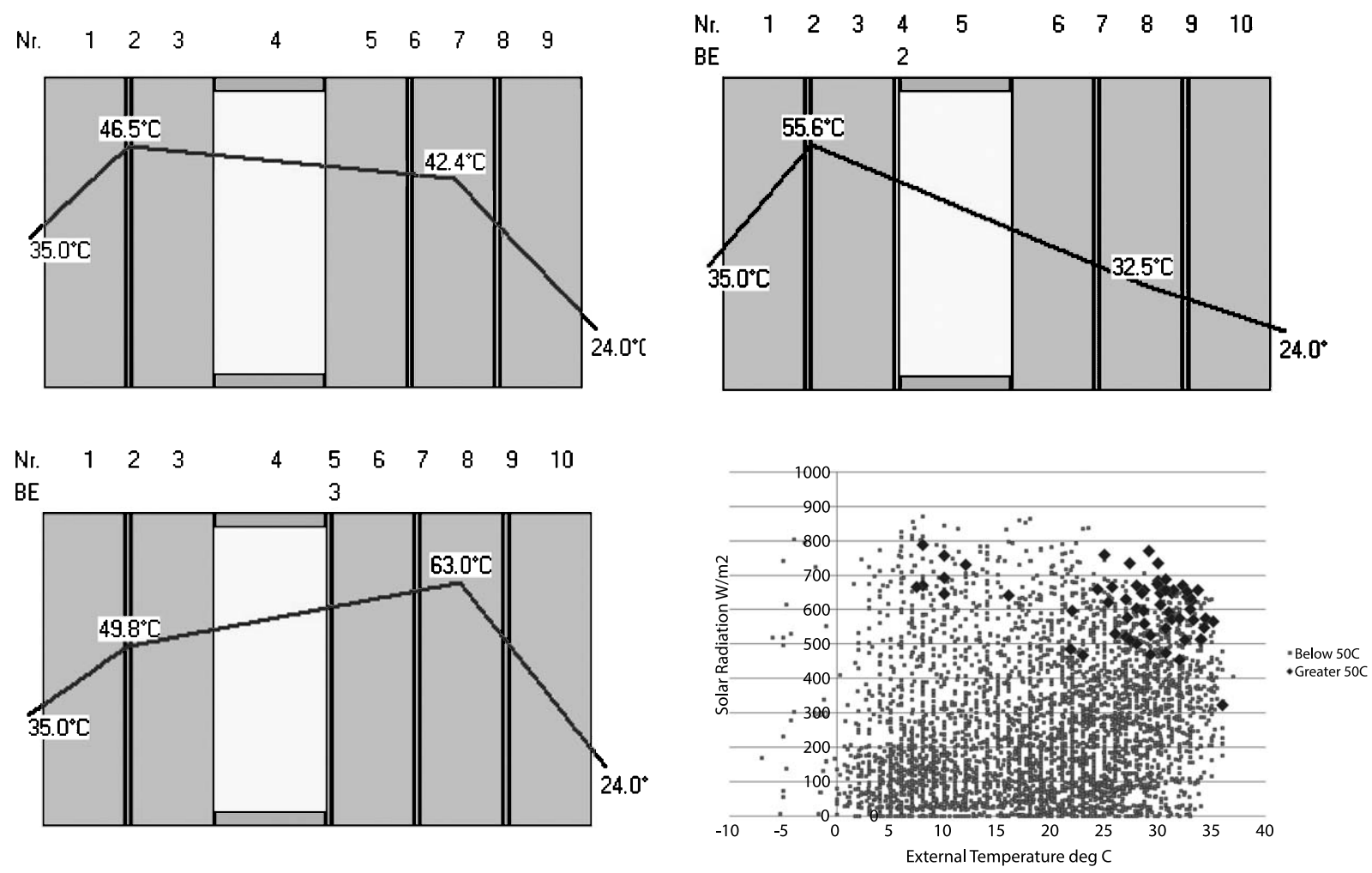

Fig. 2. Temperature division through a clear glass build-up (top left), with high performance coating on pos. 2 (top right) and with low e coating on pos. 3 (bottom left) in WinSLT, Distribution of temperatures above and below $50^{\circ} \mathrm{C}$ for the high performance coating option.

for highly exposed locations are chosen and analysed in greater detail. Figure 2 shows three of the analysed build-ups with temperature distribution due to the lower absorption compared to coated glass; the clear build-up on the top left shows the lowest temperature distribution and has been used as a benchmark as it does not meet the performance requirements. The low e coating (Fig. 2, bottom left) leads to significantly larger temperatures on the internal panes of the IGU due to the location of the coating and the increased absorption due to a thicker glass build-up. The high performance coating indicated on the top right leads to a temperature increase in the outer laminate, however is less critical than the previously mentioned option.

The data in Figure 2, bottom right, show a distribution of temperatures above and below $50^{\circ} \mathrm{C}$ occurring for the build-up using the high performance coating. This threshold has been chosen due to the significant step change in shear capacity of SGP interlayer at this temperature.

The trend clearly shows that temperatures above $50^{\circ} \mathrm{C}$ occur simultaneously with greater exterior temperatures, however, high radiation in colder months can also lead to high temperatures within the Interlayer of the laminate. For the particular location and glass configuration described in this study, the annual time with temperatures above 50 degrees is 62 hours.

For PVB and SGP interlayers, which are most commonly used today, the material stiffness changes with temperature and load duration. Through the use of viscoelastic structural analysis the designer can optimise the glass thickness in laminates if temperature variation over time can be determined 
precisely. This is crucial to a more optimised design. As the composite action with a viscoelastic interlayer increases the structural efficiency of glass laminates and reduces their weight and thickness. Furthermore, in particular for horizontal applications it is crucial that the design temperatures are known to great detail as the failure of the interlayer could lead to either strength or serviceability failure of the panel.

\section{Structural behaviour of SGP laminated glass under transient temperature loads}

Having established a set of site-specific temperature data for the part of the glass envelope of interest, a procedure for structural investigation is used herein. The transient state of the body temperature of the glass laminate, could lead to a number of challenges in the structural design. Often during the design process, the impact of this transient nature of the laminate behaviour is overlooked. This part of the study investigates SentryGlass Plus (SGP) laminated glass elements under varying temperature conditions. Due to the low transition temperature, such materials could change from solid state into semi-viscous state during the structure's lifetime or under elevated thermal conditions. Induced shear forces acting over longer time periods will cause viscous flow and therefore shear deformations.

A few examples of structural challenges related to the thermal and viscoelastic behaviour of laminated glass are presented below:

- Increased temperature gradient within a glass element may introduce significant thermal stresses. When thermal tension stresses exceed the glass edge strength, micro flaws will propagate and cause failure. In Vanderbroek, Belis, \& Louter, (2013) the authors presented a study where the thermal glass stress was calculated based on real climatic data. Yearly temperature maxima over twenty years were considered. The effect of the number of stress cycles on the fatigue strength of glass components and especially their connections should be studied further. Analysing day/night temperature gradients predominantly in climates susceptible to higher temperature variations needs extra attention.

- A decrease in the stiffness of the interlayer at an elevated temperature could shift the behaviour of the laminated glass component from monolithic to layered behaviour. Hence, a significant increase in glass bending stresses will be expected. Similarly due to the reduction in lateral stiffness, the elements could be susceptible to Euler buckling or lateral torsional instability. In Galuppi, Royer-Carfagni (2014) the authors presented a study of a laminated beam in bending and buckling where the interlayer was considered to be viscoelastic. The authors concluded noteworthy differences between the actual laminated glass beam response and the one evaluated through the quasi-elastic approximation.

- Due to the significant decrease in the bending stiffness at elevated temperatures, a laminated curved panel could be susceptible to snap through instability. Preventing abrupt global stability failure and establishing element capacity is critical as higher safety factors are usually associated with such sudden failure modes. The snap through effect of monolithic glass sheets was extensively researched in Galuppi, Massimiani, \& Royer-Carfagni (2014), where the authors investigated whether the global stability of the cold-bent panel could be enhanced by stiffening the glass edges with a metal frame bonded with soft adhesive. The authors proved by calculations that the soft adhesive distributes forces across the length rather uniformly. In Fildhuth et al. (2014) the authors presented a detailed numerical and experimental study of the recovery behaviour of laminated 
cold-bent glass. After initial short-term spring back (shape loss up to $20 \%$ ), only marginal shape loss was measured in long term for SGP laminates. However, further studies are required to investigate whether such elements, subjected to temperature cycles, especially in applications without mechanical clamping, could preserve required shape.

- Increased deformations of glass components may relate to undesirable reflections and caustic. Ponding of rainwater could increase maintenance cost as well as it might create significant aesthetic issues. Although this is primarily a serviceability and maintenance issue a possible structural problem is the increased risk of glass stress corrosion due to the water and air pollution.

\subsection{Theoretical background}

In order to determine the structural behaviour of laminated glass for a set of material properties which are time and temperature dependent, a material model is required that can accurately represent the mechanical properties of the interlayer. The investigated material is SentryGlass Plus, which falls into the category of ionomeric polymers. As such the material exhibits viscoelastic mechanical properties. A material is said to be viscoelastic if the material has an elastic (recoverable) part as well as a viscous (non-recoverable) part. Upon application of a load, the elastic deformation is instantaneous while the viscous part occurs over time (ANSYS, 2010).

The viscoelastic model usually depicts the deformation behaviour of glass or glass-like materials and may simulate cooling and heating sequences of such material. These materials at high temperatures turn into viscous fluids and at low temperatures behave as solids. Further, the material is restricted to be thermo-rheologically simple (TRS), which assumes the material response to a load at a high temperature over a short duration is identical to that at a lower temperature but over a longer duration.

Methods to define viscoelastic material model:

- Prony series

- Curve fitting

In this paper we have investigated the possibility of deriving Prony coefficients from experimental data by the curve fitting method. Experimental data were provided by the interlayer manufacturer DuPont for the SentryGlass Plus. A Dynamic Mechanical Analysis (DMA) was carried out by DuPont following the recommendations of ASTM 4065.

This test consists of applying small oscillating strain $(<10 \%)$ displacement to a tensile specimen at specified frequencies and temperatures. While the displacements are applied and loads recorded the sample compliance is determined in situ and the modulus extracted directly during the test. The tensile DMA data were analysed using the time-temperature-superposition method to extract the Young's modulus $(E)$ as a function of time for a specified reference temperature following the method described in Ferry (1980).

Curve fitting involves comparing experimental data to certain predefined nonlinear material models. Viscoelastic material curve fitting determines material constants by relating experimental data to the Prony series expansion for both the shear and bulk modulus of the hyper-viscoelastic material. After inputting the experimental data, the order of Prony series expansion is defined and a nonlinear regression is performed. The curve fitting results together with the experimental data are shown graphically in Figure 5, left. The fitted coefficients are then written to the database to define the new viscoelastic material. 


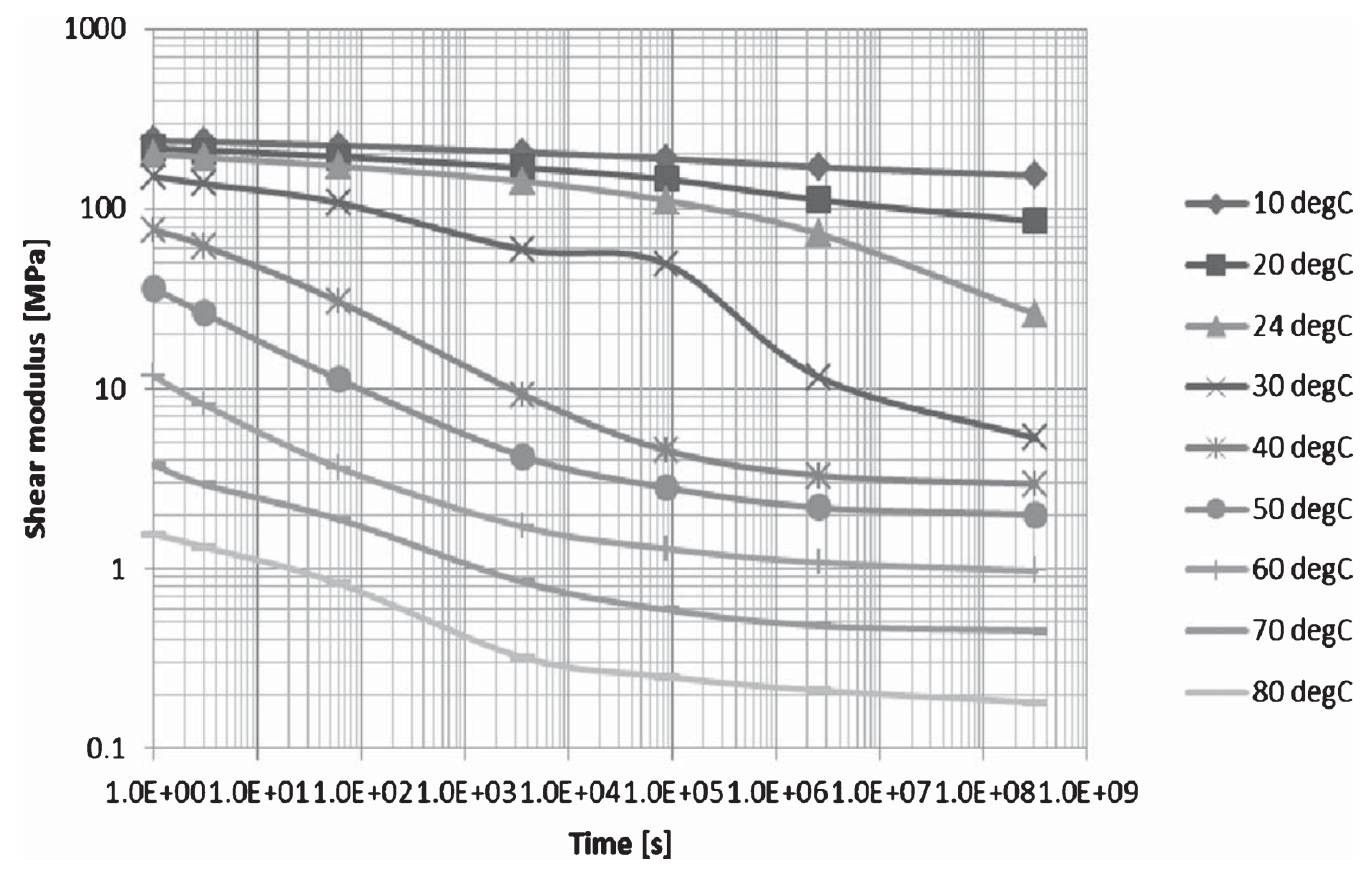

Fig. 3. Shear modulus-time /temperature dependency for Sentry Glass interlayer as published by DuPont.
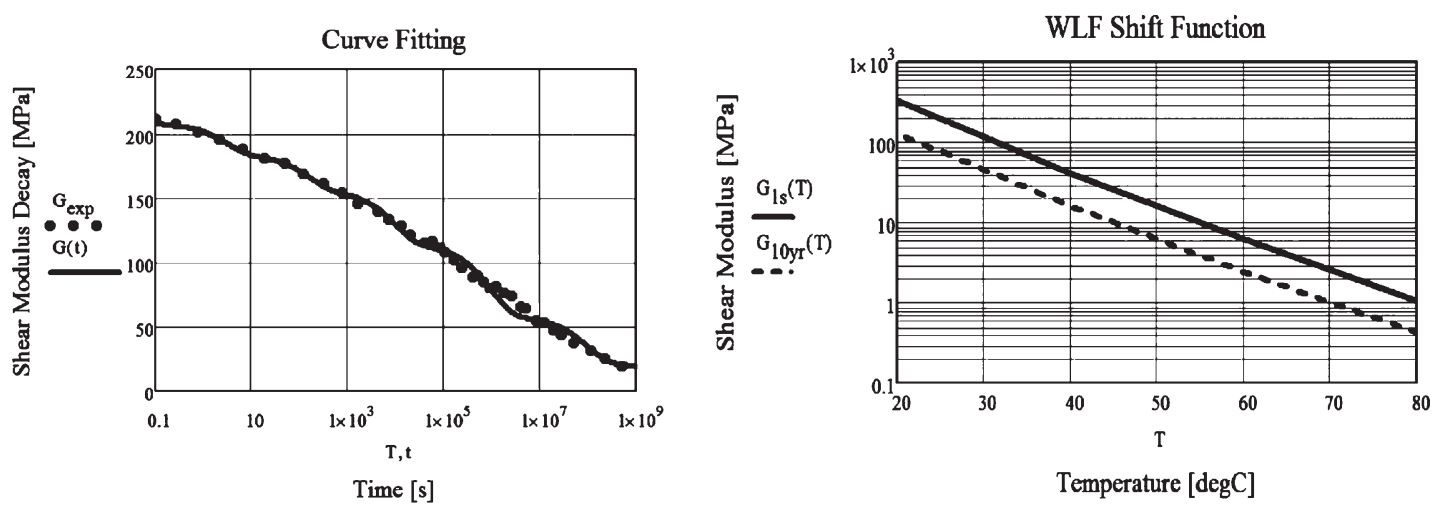

Fig. 4. Curve fitting, prony series vs. master curve of sentry glass (left) and WLF Shift function (right).

For viscoelastic curve fitting with multiple temperatures, coefficients can be evaluated at each discrete temperature point and written as a temperature-dependent Prony data table, or the WilliamsLandau-Ferry (WLF) or Tool-Narayanaswamy (TN) shift functions can be used to account for the temperature dependency. Shear modulus - time/temperature dependency for SentryGlass Plus is presented in Figure 3, while the WLF shift function is graphically displayed in Figure 4, right. 


\subsection{Numerical verification}

If the viscoelastic material is loaded with a constant force the polymer relaxes in time and strain increases with time. The corresponding shear stress decreases with time. Shear stress depends on the current strain as well as the past strain history. Due to the above effect, in general, models utilising viscoelastic materials should be stiffer than those utilising linear elastic materials which are commonly adopted in design practice.

A simple numerical experiment to test the behaviour of the viscoelastic material model was carried out. In Figure 5 the basis of the numerical model is presented. Plane stress state analysis of the interlayer region measuring $10 \mathrm{~mm}$ by $1.5 \mathrm{~mm}$ and subjected to a uniform shear load was carried out.

The specimen was loaded as shown in Figure 5, left, with constant uniformly distributed load for 5E8 seconds ( $\sim 15$ years). After that, a short rectangular impulse with constant load, 1.5 times higher than the base load, was introduced for 1E6 seconds (12 days). Then the constant base load was reapplied for another 1E9 seconds ( 30 years). The domain was meshed with eight node quadrilateral elements with initial elastic shear modulus of $200 \mathrm{MPa}$ and Poisson ratio of 0.48 . Simplified viscoelastic material modelled with Prony series for time-dependent shear modulus was used as presented in Figure 4, right.

The results from this numerical experiment are presented in Figure 7 . Three points are evaluated through the thickness $(1.0 \mathrm{~mm}, 0.5 \mathrm{~mm}$ and $0.0 \mathrm{~mm}$ from top) at the centre of the sample. In Figure 7, left, the normal stress in XX direction over time is plotted. Stress relaxation, strain flow and elastic phase followed by viscoelastic recovery are showing good correlation with theoretical viscoelastic
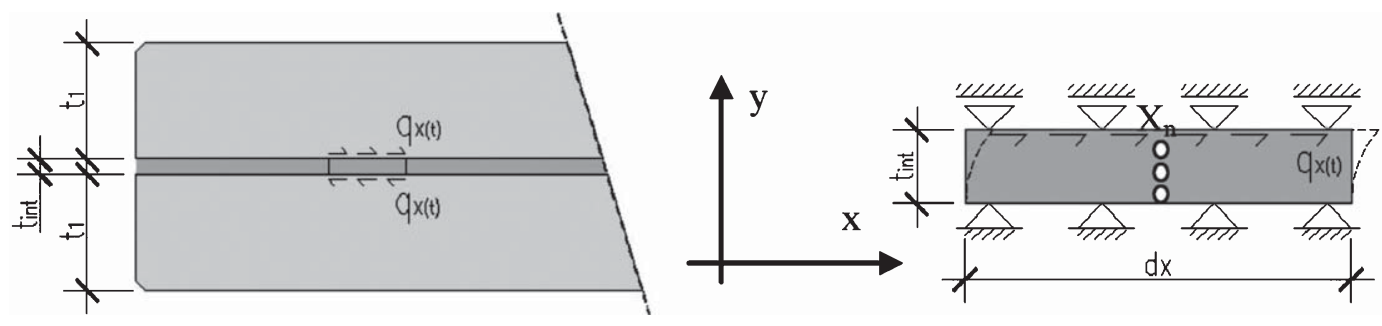

Fig. 5. Numerical experiment, definition, viscoelastic element subjected to time dependent shear.
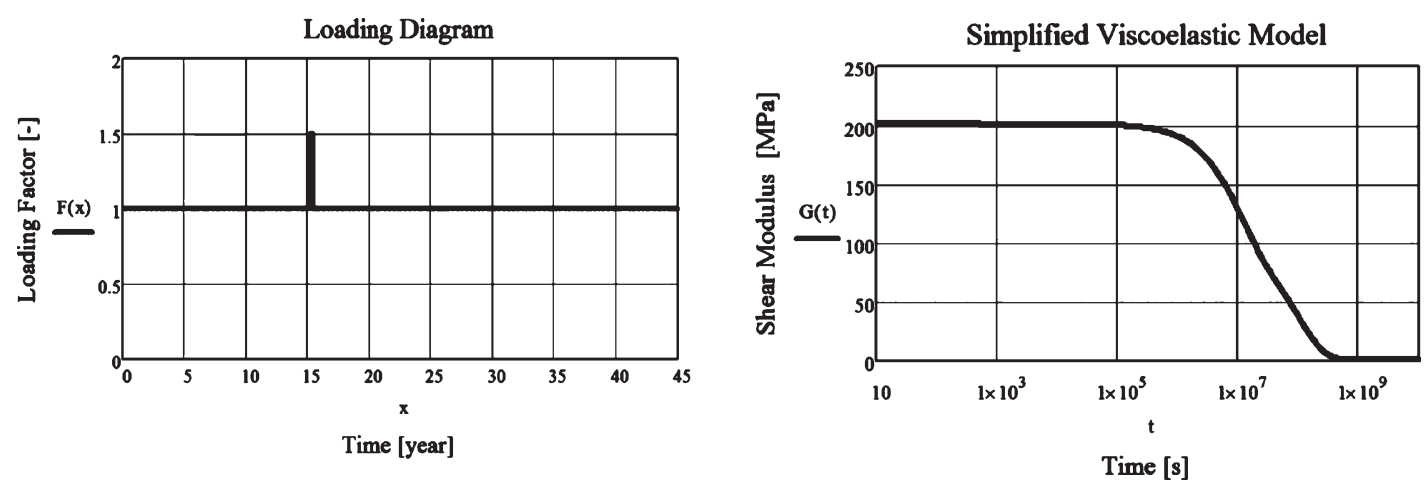

Fig. 6. Loading diagram (left) and simplified viscoelastic material model (right). 

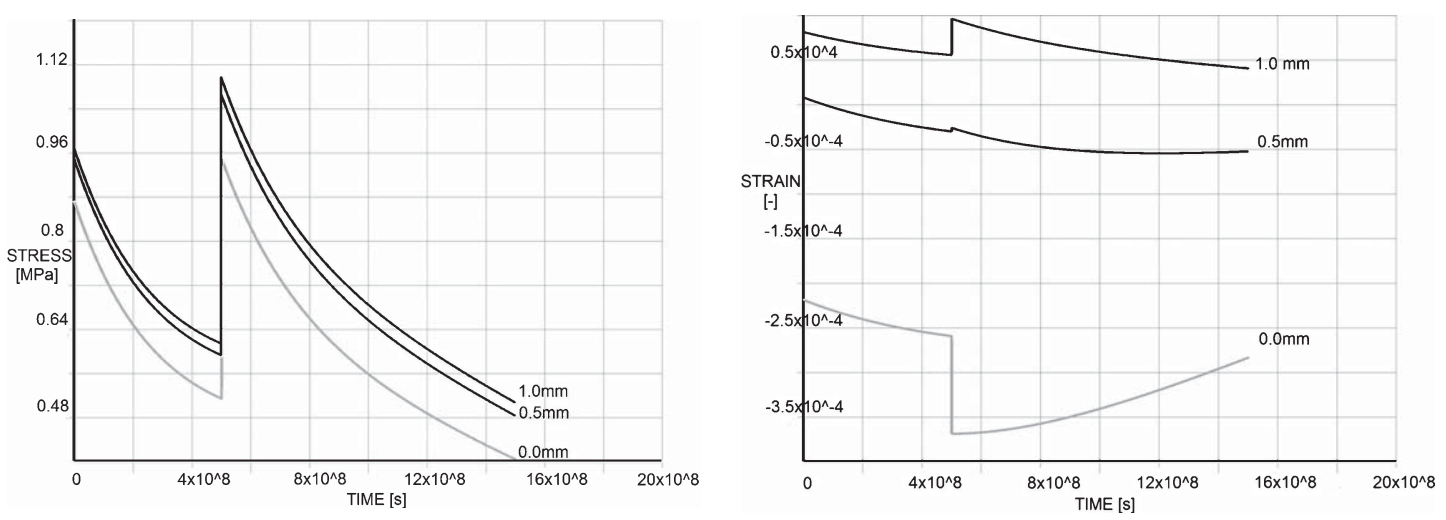

Fig. 7. Numerical experiment, Stress XX (left) and numerical experiment, Strains XX (right).

behaviour. The modelled impulse load could be replaced with a short-term temperature increase acting on the glass envelope as discussed earlier in this paper. Similar structural behaviour would be expected as materials obey TRS theorem.

To summarise, a number of techniques can be used to accurately predict the viscoelastic behaviour of the interlayer in long-term conditions. Having obtained a set of temperature data from the environmental analysis, the structural designer can use Prony series or WLF shift function to predict deflection and stress in laminated glass for a chosen set of temperature and load durations. Due to the large amount of data that are typically handled for even the simplest exercises of this sort, the authors propose the implementation of parametric tools which help to automate the processes.

\section{Parametric implementation}

Having established a methodology for carrying out environmental and structural analysis as described in this study, the designer is faced with the task of manipulating the vast amount of data that become available at each iteration. As a typical example (Fig. 8) a glazed roof structure may have thousands of potential solutions depending on the pitch angle and number of bays. The proposed temperature analysis used weather data to determine the actual temperature of the interlayers which is then used as an input for the structural analysis. By reducing the design parameters such as roof geometry, it is possible to manually design the worst-case glass panel for this example. While this is useful in itself and qualitative selection of design options remains a fundamental part of the process, there is a potential for added value to be introduced if a multi-parameter study is carried out. A viable way to tackle this is to use a common collaboration platform where the effect of each design change can be readily interrogated and its effects understood at early stages of the process (Imbert, et al., 2012).

\subsection{Parametric environment}

Building envelope design can be described as a multi-parameter problem with a large number of competing and often contradicting requirements. The solution of such a problem can be approached through the use of parametric tools, which are already well established in the industry. Tools such 

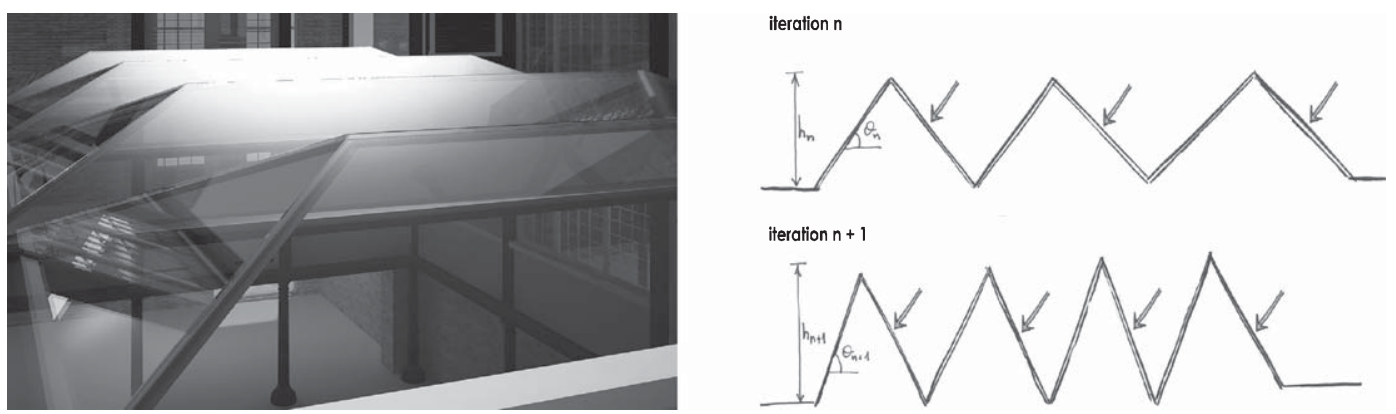

Fig. 8. Roof example (left) and incremental geometry change during optimisation (right).

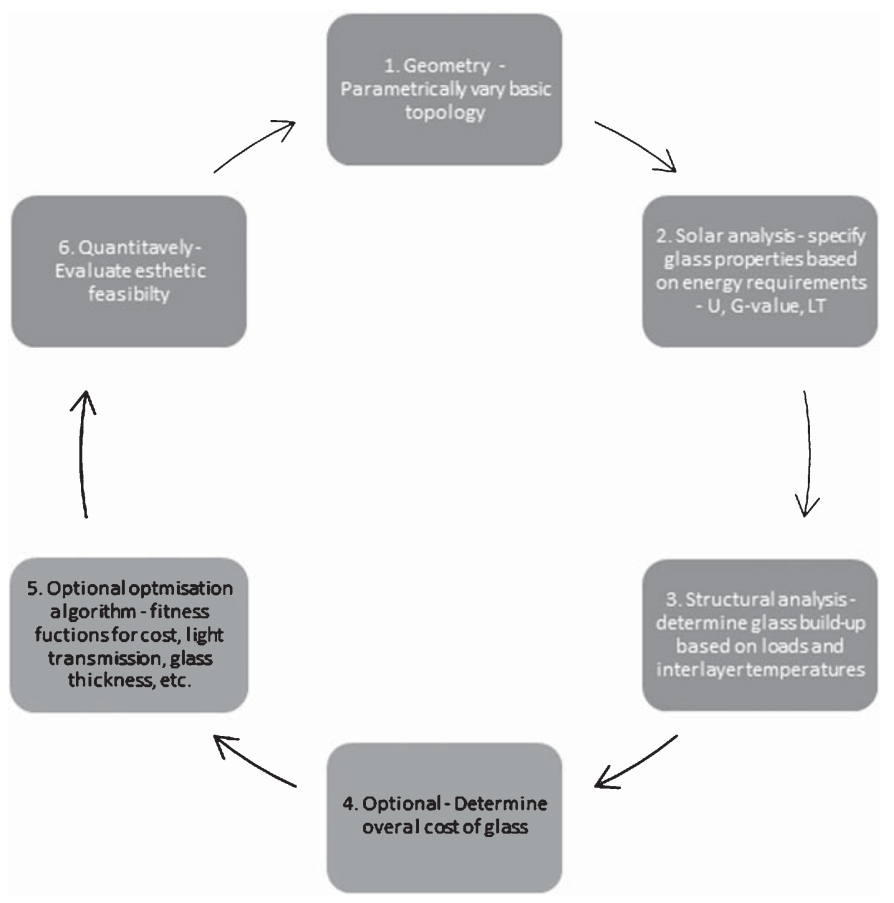

Fig. 9. Integrated parametric envelope design workflow.

as Grasshopper, Generative Components and DesignScript allow parametric design to be successfully employed in building and envelope design. In addition a large number of third-party applications for environmental and structural analysis can be integrated into the parametric environment through API scripting in one of the common languages such as $\mathrm{C}++$, Python etc. Within this parametric environment the user can dynamically explore a large number of solutions and at every step a feasibility calculation can be carried out. For instance for the roof study in Figure 8, left, we employ the process described in Figure 9.

At the first step the geometry is loosely set up by the designer, with some flexibility built into the logic (Harding, et al., 2012). Based on the available variables, e.g. pitch, ridge height (Fig. 8, right) an environmental assessment is carried out. To arrive at this, a number of tools are employed for 
environmental design, e.g. Ecotect, Diva for Rhino, which are combined with bespoke scripting to determine the amount of solar radiation and temperature within the glass build-up. The result of the assessment is a set of temperature versus time data for each glass panel centroid. In addition purpose built tools are then employed to select the thermal characteristics of the glass, i.e. whether the panels are monolithic or insulated and also have a solar coating and/or frit options. As a result the user can be informed on the current $u$, g-values and light transmission. That is then quantitatively verified against the overall building performance requirements. Following this, a structural analysis can be executed through interfacing GH with finite element software, such as Strand 7, API or ANSYS Workbench. The fore mentioned viscoelastic laminate analysis technique is then included in the parametric study. By knowing the temperature distribution in the glass build-up an accurate prediction of the glass relaxation and stresses can be calculated. All the current parameters are then fed into a budget estimate tool, which can give an approximate material cost to further inform the designer on the feasibility of the solution.

The next step in the process employs an optimisation routine, which can deliver a more structured approach to arriving at a more optimal solution. Natural selection type techniques are available in the forms of evolutionary and genetic algorithms, which can be tailored to optimise a multi-parameter task if an appropriate set of fitness functions are chosen (Rutten, 2010).

\section{Conclusion and further research}

Building envelope physics play an increasing role in the design of structural glass envelopes and are analysed in greater detail to ensure occupant comfort. This analysis today is mainly performed by building physics experts or mechanical engineers. As such there is little or no information being passed between these teams and the structural designer. The authors see this as a vital link which, if utilised, can increase the performance and reduce the cost of envelopes. The study presented here proposes a methodology for tackling this challenge. The information gained by the environmental analysis, more precisely the temperature variation of the interlayer over time, is used as an input to nonlinear structural analysis. In this paper only a single short term peak temperature load (12 hour) was analysed with a base temperature over a total duration of 45 years. The aforementioned numerical experiment was carried out to test the stability of the numerical analysis as well as the behaviour of the proposed viscoelastic material model. While this proves the feasibility of the approach the authors acknowledge that carrying out visco-elastic analysis coupled with time dependent load curves is computationally highly expensive. It is acknowledged that analysis of such dimension requires an automated procedure for handling the data. As such a methodology has been presented for the implementation of a parametric system which is capable of iterating through a large number of solutions without the need for manual user input.

Further research into this topic will be beneficial as relates to the analysis and material models available for the simulation of ionomer interlayers as well as other commercially available laminating substrates. The authors acknowledge the limitations of the WLF shift function as an accurate representation of the SGP behaviour but have included it for completeness. In line with the proposed numerical verifications, small scale physical testing is required which extends into a more realistic time span than the results currently available in the field, e.g. one to five years. To achieve this, a temperature and humidity controlled environment would be beneficial in the further study of permanent and variable strain applied to the specimens. The data would prove invaluable for calibration with existing numerical models. 


\section{References}

ANSYS Inc. \& SAS IP inc. 2010, ANSYS 13.0 Theoretical Manual.

De Bleecker, H., Scholz, C., Mocibob, D., Standaert, P. (2014). A novel glass facade with high environmental targets. In: C. Louter, F. Bos, J. Belis \& J.-P. Lebet (Eds.), Challenging Glass 4 \& Cost Action TU0905 Final Conference (pp. 161-170). London, United Kingdom: Taylor \& Francis Group.

EnergyPlus Energy Simulation Software, Weather Data, WMO Region 4 - Mountain View-Moffett Field NAS 745090 (TMY). Retrieved May 1, 2014 from http://apps1.eere.energy.gov/buildings/energyplus/weatherdata_about.cfm

Ferry, J.D. (1980). Viscoelastic Properties of Polymers, 3rd edition, John Wiley \& Sons.

Fildhuth, T., Knippers, J., Bindji-Odzili, F., Baldassini, N., Pennetier, S. (2014). Recovery behaviour of laminated cold bent Glass. In: C. Louter, F. Bos, J. Belis \& J.-P. Lebet (Eds.), Challenging Glass 4 \& Cost Action TU0905 Final Conference (pp. 113-120). London, United Kingdom: Taylor \& Francis Group.

Galuppi, L., Massimiani, S., Royer-Carfagni, G. (2014). Snap-through instability in double curved cold-bent glass. International Journal of Non-Linear Mechanics, Elsevier 2014, In Press.

Galuppi, L., Royer-Carfagni, G. (2014). The design of laminated glass under time-dependent bending and buckling. In: C. Louter, F. Bos, J. Belis \& J.-P. Lebet (Eds.), Challenging Glass 4 \& Cost Action TU0905 Final Conference (pp. 431-438). London, United Kingdom: Taylor \& Francis Group.

Harding, J. (2012). Thinking Topologically at Early Stage Parametric Design, Advances in Architectural Geometry, 77-90.

Hindrichs, D., Daniels, K. (Eds.) (2007). Plusminus 20\% $40^{\circ}$ latitude. Edition Axel Menges. Stuttgart/London.

Imbert, F. (2012). Concurrent Geometric, Structural and Environmental Design: Louvre Abu Dhabi, Advances in Architectural Geometry, 67-76.

Rutten, D. (2010). Evolutionary Principles applied to Problem Solving. Retrieved May 17, 2014 from http://www.grasshopper3d.com/ profiles/blogs/evolutionary-principles

Vanderbroek, M., Belis, J., Louter, Ch. (2013). Thermal breakage of glass. In: J. Belis, Ch. Louter, \& D. Mocibob (Eds.), Cost Action TU0905 Mid-term Conference on Structural Glass (pp. 563-569). London, United Kingdom: Taylor \& Francis Group. 